\title{
Resistance integrons: class 1, 2 and 3 integrons
}

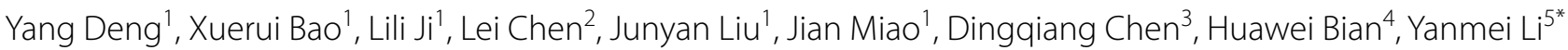 \\ and Guangchao Yu ${ }^{6^{*}}$
}

\begin{abstract}
As recently indiscriminate abuse of existing antibiotics in both clinical and veterinary treatment leads to proliferation of antibiotic resistance in microbes and poses a dilemma for the future treatment of such bacterial infection, antimicrobial resistance has been considered to be one of the currently leading concerns in global public health, and reported to widely spread and extended to a large variety of microorganisms. In China, as one of the currently worst areas for antibiotics abuse, the annual prescription of antibiotics, including both clinical and veterinary treatment, has approaching 140 gram per person and been roughly estimated to be 10 times higher than that in the United Kingdom, which is considered to be a potential area for the emergence of "Super Bugs". Based on the integrons surveillance in Guangzhou, China in the past decade, this review thus aimed at summarizing the role of integrons in the perspective of both clinical setting and environment, with the focus on the occurrence and prevalence of class 1,2 and 3 integrons.
\end{abstract}

Keywords: Antimicrobial resistance, Mobile genetic elements, Horizontal transfer, Resistance integrons

\section{Background}

Antibiotics, as compounds or substances that kill or inhibit the growth of microorganisms, have been regarded as one of the greatest contributions to medicine and humanity in the 20th century and used to treat a wide range of infectious diseases caused by bacteria, for both animals and human beings [1-4]. However, as recently indiscriminate abuse of existing antibiotics in both clinical and veterinary treatment leads to proliferation of antibiotic resistance in microbes and poses a dilemma for the future treatment of such bacterial infection, antimicrobial resistance has been considered to be one of the currently leading concerns in global public health, and reported to widely spread and extended to a large variety of microorganisms, which will consequently result in an increasing number of clinical failures in bacterial mediated diseases [2, 3, 5]. A number of resistance mechanisms are responsible for the emergence and

\footnotetext{
*Correspondence: yanmeili@live.cn; guangchaoyu@126.com

${ }^{5}$ Guangzhou Women and Children's Medical Center, 9 Jinsui Road, Guangzhou 510620, China

${ }^{6}$ First Affiliated Hospital of Jinan University, Guangzhou 510620, China Full list of author information is available at the end of the article
}

prevalence of antimicrobial resistance, and such mechanisms have been divided into genetic mutation occurred at a low frequency and acquisition of various genes mediated resistance to their host microorganisms. As consequence, acquisition of resistance genes has been regarded as major contributor for the wide distribution and spread of antimicrobial resistance, via either vertical transfer and horizontal transfer, with the latter mechanism involving mobile genetic elements such as plasmids and transposons [3]. As mostly carried by plasmids or contained within a transposon, integrons as well as its mechanism and role played in the distribution of microorganisms have been well established and documented [6, 7], which had also been considered to contribute to the unleashing of "Super Bugs" [3, 8]. In China, as one of the currently worst areas for antibiotics abuse, the annual prescription of antibiotics, including both clinical and veterinary treatment, has approaching 140 gram per person and been roughly estimated to be 10 times higher than that in United Kingdom [3, 8, 9].

Since the first report in 1989 [10], the molecular mechanism and mobility of integrons, including the excision and integration for gene cassettes, had been investigated

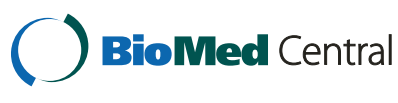

(c) 2015 Deng et al. This article is distributed under the terms of the Creative Commons Attribution 4.0 International License (http://creativecommons.org/licenses/by/4.0/), which permits unrestricted use, distribution, and reproduction in any medium, provided you give appropriate credit to the original author(s) and the source, provide a link to the Creative Commons license, and indicate if changes were made. The Creative Commons Public Domain Dedication waiver (http://creativecommons.org/ publicdomain/zero/1.0/) applies to the data made available in this article, unless otherwise stated. 
and validated for the following years $[6,7,11]$. Moreover, its occurrence in clinical microorganisms and its role played in antimicrobial resistance were also widely studied for the past decades $[12,13]$.

\section{Structure}

An integron is generally defined by the presence of an integrase gene (intl) and a proximal primary recombination site (attI) (Fig. 1) [2, 14]. The amino acid sequences of IntI integrases have been used as a basis for dividing integrons into 'classes', with those carrying intI1 defined as 'class 1', intI2 as 'class 2', intI3 as 'class 3', etc. intI1, intI2 and intI3 were first identified in association with mobile genetic elements and intI4 and others with chromosomal integrons. As the most commonly selected target for the detection of an integron, intI encodes an integrase (IntI) of the tyrosine recombinase family, which is characterized by the distinct presence of invariant RHRY (with $\mathrm{Y}$ being the catalytic tyrosine) amino-acids in the conserved motifs called box 1 and box 2 that discriminate intI within the other XerC-related integrase) [2]. IntIcatalysed recombination between attI and/or attC sites results in insertion or excision of cassettes (Fig. 1). The class 1 integrase (IntI1) recognises three types of recombination site: attI1, attC and secondary sites. Binding

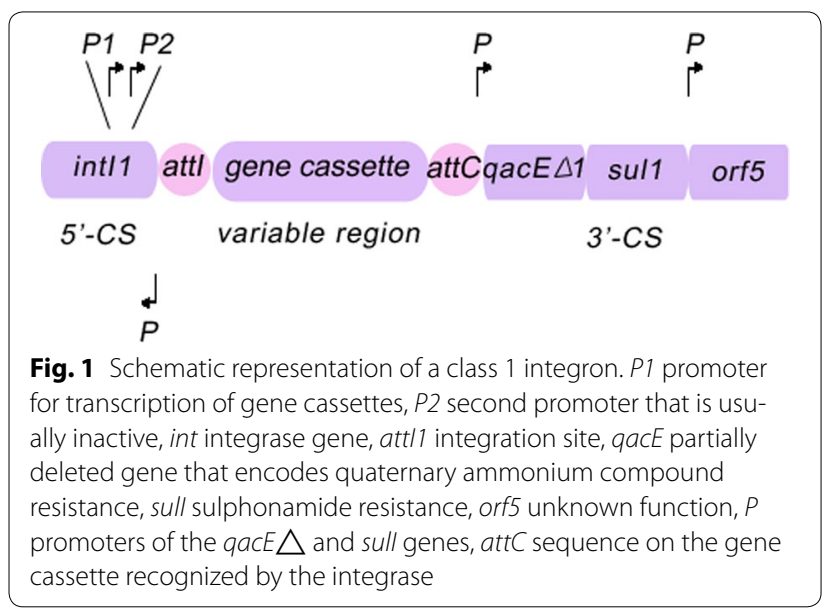

domains and consensus sequences have been determined for these. The attI1 site is a simple site which contains two inverted sequences that bind the integrase, and two additional integrase-binding sites known as strong (DR1) and weak (DR2) (Fig. 2) [11, 15].

\section{The attC sites}

The attC region contains two simple sites, each composed of a pair of conserved 'core sites' (7 or $8 \mathrm{bp}$ ), referred to as $\mathrm{R}^{\prime \prime}$ and $\mathrm{R}^{\prime}, \mathrm{L}^{\prime}$ and $\mathrm{L}^{\prime \prime}$ [2]. The $\mathrm{R}^{\prime}$ and $\mathrm{R}^{\prime \prime}$ sites are part of the $\mathrm{RH}$ consensus sequence, which is more or less equivalent to the $\mathrm{RH}$ simple site. The $\mathrm{L}^{\prime}$ and $\mathrm{L}^{\prime \prime}$ sites are part of the $\mathrm{LH}$ consensus sequence, which is more or less equivalent to the $\mathrm{LH}$ simple site $[8,11]$. The $\mathrm{LH}$ and $\mathrm{RH}$ sites in the attC are possibly distinguished by the integrase, which might explain the orientation of integration of the gene cassettes. L" also appears to be significant for orientation [15]. The LH simple site is not only required for orientation but also enhances $\mathrm{RH}$ activity $[16,17]$. The attC sites are generally associated with a single ORF in a structure termed gene cassettes, which are not necessarily observed in integrations, but once integrated they become part of the integron [11].

\section{Gene cassettes}

According to previous reports, cassettes located within the variable region of integrons are sometimes absent in the structure of integrons [18]. Via specific excision and integration, gene cassettes are integrated between two recombination sites (attI and $a t t C$ ) and thus become part of the integron, and exist in either the independent circular DNA molecule which is unable for stably maintain during cell division or the linear form which is created by a highly orientation-specific insertion of the free circular element into the integron [2]. Despite possession of a coding sequence, gene cassettes are generally found to be lack of promoters to constitute the mobile component of the system, and most cassettes encode resistance against antibiotics cover a wide range of antibiotics, with up to date more than 130 distinct antibiotic resistance genes characterized via unique attC sites [19, $20]$. Together, these cassettes confer resistance to most classes of antibiotics containing all known $\beta$-lactams,

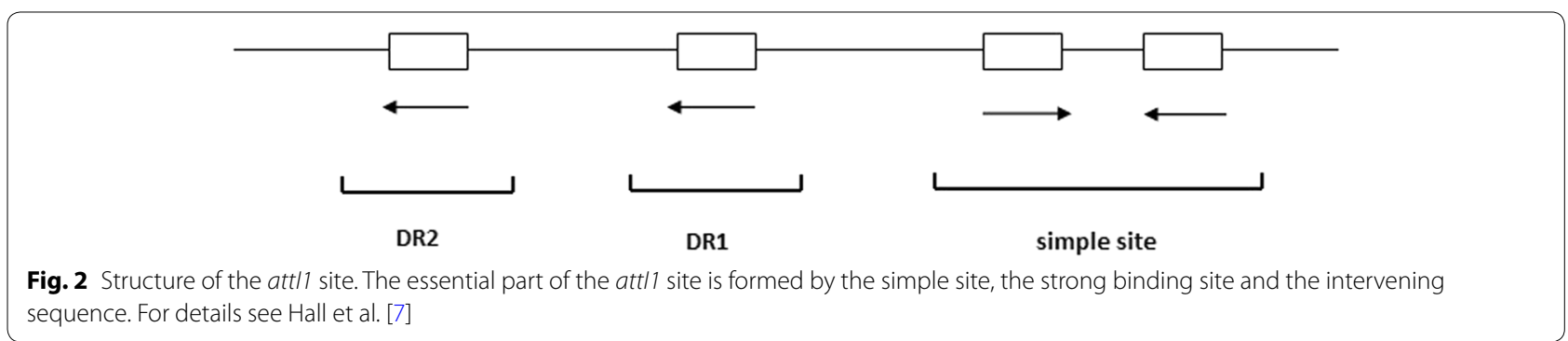


all aminoglycosides, chloramphenicol, streptothricin, trimethoprim, rifampin, erythromycin, quinolones, fosfomycin, lincomycin, and antiseptics of the quaternary ammonium-compound family [21,22].

\section{Mobility}

Reported as widely spread and distributed in clinical organisms, the mobility of integrons has been considered to be a major concern of clinically antibiotic resistance, which is defined as being associated with mobile DNA elements (transposons or plasmids) and antibioticresistance genes in addition to having a small array size and substantial heterogeneity in the sequence of attC sites [7, 13]. Despite the defectivity of self-transposition, currently existent integrons (mostly class 1 integron) has been considered to be a potentially mobile genetic element and commonly found to be located on plasmids as facilitation of conjugative-mediated transfer, as it contains gene cassettes that are mobile and capable of transferring to other integrons or to secondary sites in the bacterial genome. The integron system is a natural capture system and assembly platform, which allows microorganisms to incorporate gene cassettes and further convert them to functional proteins via correct expression. Each unique ORF is conceivably capable of being structured as a novel type of gene cassette and vital to decipher the mechanism governing cassette genesis. As a consequence, with the naturally huge pool of gene cassettes, integron may have the potentially limitless capacity to exchange and stockpile functional gene cassettes which consequently permits rapid adaptation to selective pressure and may ultimately endow increased fitness and advantage to the host $[7,13]$. In addition, mobile genetic elements, including conjugative plasmids, transposons, insertion sequences and genomic islands, may potentially be the vast reservoirs and massive genetic pool for integron, which will further be shared among bacteria [20, 23]. With mobility from gene cassettes, integrons play key role in the dissemination and spread of resistance genes, responsible for both spread and exchange of resistance genes to a wide range of distinct antibiotics among diverse bacteria [23, 24]. Aside from clinical perspectives, a large number of reports on integrons from environmental microorganisms, as well as the high sequence diversity observed and various functional products other than resistance encoded by such cassettes, strongly indicates integrons are ancient genetic element within the genomes and may have played a critical role in evolution and adaptation for a considerable period [25].

\section{Classification}

From the differences and divergence in the sequences of intI, integrons have been classified and divided into several classes. Up to date, 4 general classes of integrons have been identified and distinguished, termed classes 1-4 integrons. Known as multi-resistant integron (RIs), classes 1-3 integrons are capable of acquiring same gene cassettes via similar recombination platform, which had been supported by the in vitro excision and integration occurred via recombination sites from such integrons [11]. Most of the currently available studies on integrons had been conducted on class 1 integron, with focus on Gram-negative microorganisms. As a distinct type of integron, class 4 integron was firstly identified on the small chromosome of Vibrio cholerae and found to be an integral component of many $\gamma$-proteobacterial genomes $[26,27]$, which had also been considered to be a leading concern on both antimicrobial resistance and bacterial genome evolution, despite the limitation of the associated reports within the species of Vibrio. The remaining classes of integrons may also contain antibiotic resistance gene cassettes, but their worldwide prevalence remains low [28].

\section{Class 1 integron}

Integrons have been found in approximately $9 \%$ of the sequenced bacterial genomes, and class 1 integron platform is the most ubiquitous and has been the most commonly reported among clinical bacteria and remains the focus of numerous studies [29, 30]. Considered to be directly linked with Tn402-like transposons and associated with $\operatorname{Tn} 3$ transposon family ( $\operatorname{Tn} 21$ or $\operatorname{Tn} 1696)$, class 1 integron is not self-movable, while other mobile genetic elements such as conjugative plasmids and transposons associated are able to serve as vehicles for the intraspecies and interspecies transmission of genetic material through site-specific recombination reaction mediated by either the Tn21 integrase or the integron integrase IntI1 when the integration sites conform to the consensus sequence GWTMW or GNT (Fig. 3), respectively [20, 31]. Three types of recombination sites (attI1, attC and secondary sites) are able to be recognized by intI1, though with different recombination efficiency as recombination event between attI1 site and $a t t C$ has been shown slightly more efficient than recombination between two attC sites, and that between two attI1 sites far less efficient, with recombination by secondary sites with attC more efficient than that with attI. As a consequence, this class of integron is capable of capturing gene cassettes via this site-specific recombination platform, and gene cassettes are also able to be further expressed from a common promoter located in the $5^{\prime}$-conserved segment $\left(5^{\prime}\right.$-CS) region where two potential promoter sites Pc (also known as $\mathrm{P}_{\mathrm{ANT}}$ ) and P2 locate, with Pc approximately 200 bp upstream of the integration site [25]. Despite the dispensability for the site-specific recombination platform, Pc plays a key 


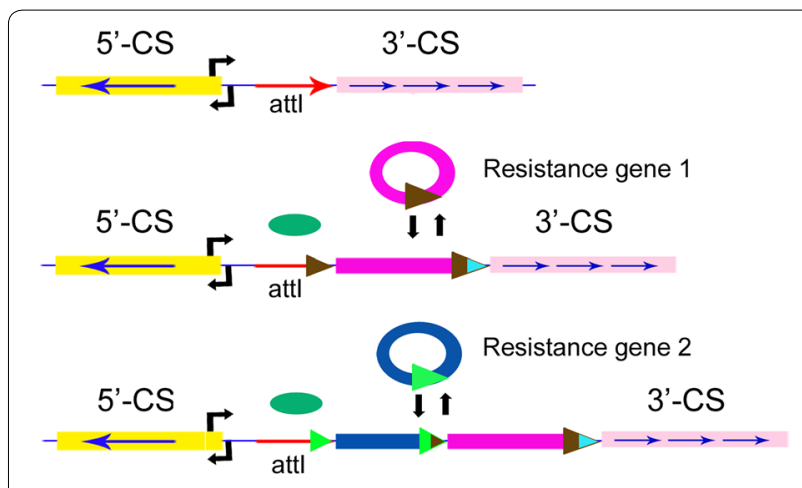

Fig. 3 Integration and excision of gene cassettes by site-specific recombination. Intl encoded by the int/ gene in the integron catalyses recombination between the att/1 site of the integron and/or the attC site(s) of gene cassette(s) resulting in insertion or excision of a cassette. One or more noncassette resistance genes may be inserted at the position of the $3^{\prime}$-CS. Horizontal arrows indicate the opposite orientations of intl and cassette-borne genes

role in the functioning of integron as it ensures the correct expression of gene cassette, as comparatively P2 is inactive as the replacement of the optimal 17 nucleotides between the -35 and -10 boxes to only 14 nucleotides [25]. Downstream of gene cassette within a typical class 1 integron, the $3^{\prime}$-conserved segment ( $3^{\prime} \mathrm{CS}$ ) possesses the genes qacE $\Delta 1$ and sul1, encoding resistance to quaternary ammonium salts and sulfonamide, respectively
[31]. Escherichia, Pseudomonas, Salmonella, Staphylococcus, Enterococcus and Vibrio have still been considered to be frequent pathogens responsible for various bacterial infections and diseases [2, 3, 32-36], and their relevant chemotherapy are clinically significant. As a common contributor to the wide distribution and spread of antimicrobial resistance, class 1 integron has been studied in various microorganisms, with its occurrence and prevalence commonly reported to be ranging from 22 to $59 \%$ and identified in clinical Gram-negative bacteria, including Acinetobacter, Aeromonas, Alcaligenes, Burkholderia, Campylobacter, Citrobacter, Enterobacter, Escherichia, Klebsiella, Mycobacterium, Providencia, Pseudomonas, Salmonella, Serratia, Shigella, Stenotrophomonas, and Vibrio (Table 1) [3, 14, 16-20, 23, 32-48]. As the local studied area was concerned, class 1 integrons were commonly found in Gram-negative bacteria isolated in Guangzhou, southern China during 2001-2006 [2, 49], with an occurrence of $73.6 \%$ (243/330), with high prevalence for E. coli, K. pneumoniae, Acinetobacter spp. and Enterobacter cloacae, except for P. aeruginosa (45.8\%, 54/118).

Class 1 integron has been well established and documented in Gram-negative microorganisms, with its role in the distribution and spread of antimicrobial resistance also verified and identified. Class 1 integrons are associated with a variety of resistance gene cassettes, but most integrons contain an aadA resistance determinant,

Table 1 Occurrence and prevalence of class 1 integron in Gram-negative microorganisms

\begin{tabular}{|c|c|c|c|c|}
\hline Date & Bacterial & $\begin{array}{l}\text { Occurrence of class } 1 \text { integron } \\
\text { and the array of gene cassettes }\end{array}$ & Sampling & References \\
\hline 2006 & Shigella & EstX-aadA1 $(3.85 \%, 1 / 26)$ & Hiroshima prefecture, Japan; 2000-2004 & {$[34]$} \\
\hline 2002 & Salmonella & $\begin{array}{l}36.2 \% \text { (34/94); aadA2-bla (PSE-1) }(61.76 \% 21 / 34) \\
\text { aadA1-aadA2-bla (PSE-1) }(38.23 \%, 13 / 34)\end{array}$ & Animals, Japan & {$[33]$} \\
\hline 2000 & V. cholerae & 44/176; aadB-aadA2-blaP1-dfrA1-dfrA15 & Thailand & {$[39]$} \\
\hline 2002 & Burkholderia & $29.4 \%(5 / 17) ;$ oxa-aac (6'-1a) & Ireland & {$[38]$} \\
\hline 2004 & Campylobacter & $62 / 378$ & Ireland & {$[37]$} \\
\hline 2008 & Enterobacteriaceae & $50 / 226$ & Addenbrooke's Hospital & {$[62]$} \\
\hline 2005 & Escherichia coli & 4/32 (12.5\%); sat-1-aadA & Meat and meat products, Norwey & {$[42]$} \\
\hline 2008 & E. coli & $59.5 \%(355 / 597)$ & South Thailand & {$[65]$} \\
\hline 2011 & E. coli & & Preliminary study in Guangzhou, China & {$[3]$} \\
\hline 2009 & P. aeruginosa & $45.8 \%(54 / 118)$ & Preliminary study in Guangzhou, China & {$[19]$} \\
\hline 2008 & Serratia & 1/30; aacC1-ORFX-ORFY-aadA1 & Canada & {$[17]$} \\
\hline 2004 & Stenotrophomonas maltophilia & $22 \%(20 / 93)$ & Kaohsiung Medical University & {$[36]$} \\
\hline 2013 & P. aeruginosa & $43 \% 37 / 182$ & Guilan, Iran & {$[44]$} \\
\hline 2011 & K.pneumoniae & $18 / 26$ & Blood stream infections & {$[2]$} \\
\hline 2013 & S. enteritidis & $11.9 \%(59)$ & Taiwan & {$[41]$} \\
\hline 2013 & S.panama & $40.0 \%(20)$ & Taiwan & {$[41]$} \\
\hline 2010 & P. aeruginosa & High prevalence & Iran & {$[40]$} \\
\hline 2009 & Aeromonas & 16/41 (39.02 \%); dfrA15-cm/A4-aadA2 & Hidalgo, Mexico & {$[32]$} \\
\hline
\end{tabular}


encoding streptomycin-spectinomycin resistance. Trimethoprim resistance determinants are also detected frequently $[12,21,22]$. This is not surprising because trimethoprim + sulphamethoxazole has been a therapeutic combination used frequently [12]. Class 1 integrons isolated from bacteria involved in infections of man frequently also harbor gene cassettes encoding $\beta$-lactam resistance [22]. In addition, new gene cassettes encoding resistance against these aminoglycosides have been discovered during the last few years [45]. However, such studies have been significantly restricted to species of Gram-negative bacteria, with only a few examples amongst Gram-positive organisms. Up to date, class 1 integrons have been reported on Gram-positive bacteria including Corynebacterium, Streptococcus, Enterococcus, Staphylococcus, Aerococcus and Brevibacterium, and gene cassettes $a a d A$ and $d f r A$ were most frequently detected (Table 2). In 1998, the first evidence of class 1 integron among Gram-positive bacteria was reported as the complete class 1 integron was detected on a $29-\mathrm{kb}$ plasmid pCG4 associated streptomycin/spectinomycin resistance determinant from Corynebacterium glutamicum [50]. In 1999, aadA (an integron-related gene) was recovered in E. faecalis strain W4470, with the transfer of class 1 integron via a plasmid between $E$. faecalis of the horizontal transfer [51]. In 2002, an intI1-like gene truncated by $I S 6100$ was found on a 27.8 -kb R-plasmid pTET3 in C. glutamicum LP-6, which mediated resistance to streptomycin, spectinomycin and tetracycline [23]. During 2001-2004, a total of 15 enterococcal strains isolated in Guangzhou, China were detected to be positive for class 1 integrase and $3^{\prime}$-conserved region of qacE $\Delta 1$-sul1, with class 2 integrons also discovered in two E. faecalis strains [52]. During 2001-2002, class 1 integrons had been detected in four consecutive Streptococcus strains sampled from First Affiliation Hospital of Jinan University in Guangzhou, China, with an array of dfrA12-orfF-aadA2 [52]. In 2004, class 1 integrons were recovered from several species of Corynebacterium spp., (C. ammoniagenes, C. casei and C. glutamicum), Aerococcus spp., Staphylococcus and Brevibacterium thiogenitalis from poultry litter [43]. As Staphylococcus strains are considered to be the top three contaminating pathogens (with HBV and HIV) [51], the finding of class 1 integrons in this genus from the recent decade is notable. During 2001-2006, class 1 integrons were commonly found in clinical Staphylococcus isolated from FAHJU and Guangdong Provincial People's Hospital in Guangzhou, China. Within this integron investigation conducted in Guangzhou, class 1 integrons were detected in 122 MRS strains (from 262 MRS isolates, with 209 MRSA and 53 MRCNS); no class 2 or 3 integrons were obtained [2, 3, $49,53]$. In 2009, class 1 integron was identified from one
S. epidermidis strain isolated from Bogota, Colombia, which carried the $78 \%$ homologous int 1 and the cassette arrays of aac6 (aminoglycoside acetylation) with resistance to aminoglycoside and $a a c 6^{\prime}$-aph $2^{\prime}$ with resistance to $\beta$-lactams [54]. In 2013, class 1 integrons was reported on 81 Staphylococcus isolates $(40.5 \%, 81 / 200)$ recovered from nasal and throat swabs in Sanandaj Hospital, Iran, including 37 (40.1\%) S. aureus, 35 (23.5\%) S. epidermidis and 9 (36.0 \%) S. saprophyticus strains [55].

\section{Class 2 integron}

Similar to the organization of class 1 integron, class 2 integron is commonly found to be associated with the Tn7 transposon family ( $\operatorname{Tn} 7$ and its derivatives, such as $\operatorname{Tn} 1825, \operatorname{Tn} 1826$ and $\operatorname{Tn} 4132$ ), carrying both of its recombination site attI2 and promoter Pc found within such transposons [19]. Its $3^{\prime}$ conserved segment (3'-CS) contains 5 tns genes (tnsA, tnsB, tns $C$, tns $D$ and tnsE) functioning in the movements of transposon [56], which mediates the mobility of class 2 integron via a preferential insertion into a unique site within bacterial chromosomes [30,57]. The homology of amino-acid sequences of a typical intI2 gene are found to be less than $50 \%$ comparing to the intI1, and unfunctional due to the replacement of the internal termination codon with glutamic acid (amino acid 179) and thus the production of a shorter and inactive polypeptide which was unable to catalyse the recombination reaction [58]. Though the origin of this stop codon still remains unclear, the two current explanations for this potentially pseudogene are available as follows: (1) the regulatory function; (2) the functioning from presence of other type of integrase (mostly intI1). Such assumption has been supported by the simultaneous carriage of class 1 and class 2 integrons, the limited number of different arrays of gene cassettes, as well as the low diversity of cassette genes obtained. Despite its capability of site specific excision and integration of gene cassettes precisely into attI2, intI2 is unable to recognize the attC sites of gene cassettes from class 1 integrons and mediate further integration. However, class 2 integrons share identical gene cassettes with class 1 integrons, such as $d f r A 1$, sat 1 and aadA1. The classic structure of class 2 integrons contain an array of gene cassettes, including dihydrofolate reductase ( $f f r A 1)$, streptothricin acetyltransferase (sat1), and aminoglycoside adenyltransferase (aadA1), which confer resistance to trimethoprim, streptothricin and streptomycin/spectinomycin, respectively $[19,57]$. However, in the past decade, novel rearrangements of cassettes and resistance genes had been reported and identified. In detail, an erythromycin esterase gene (ereA) was detected in a class 2 integron containing its own promoter and capable of being propagated by a class 2 integron with an insertion 


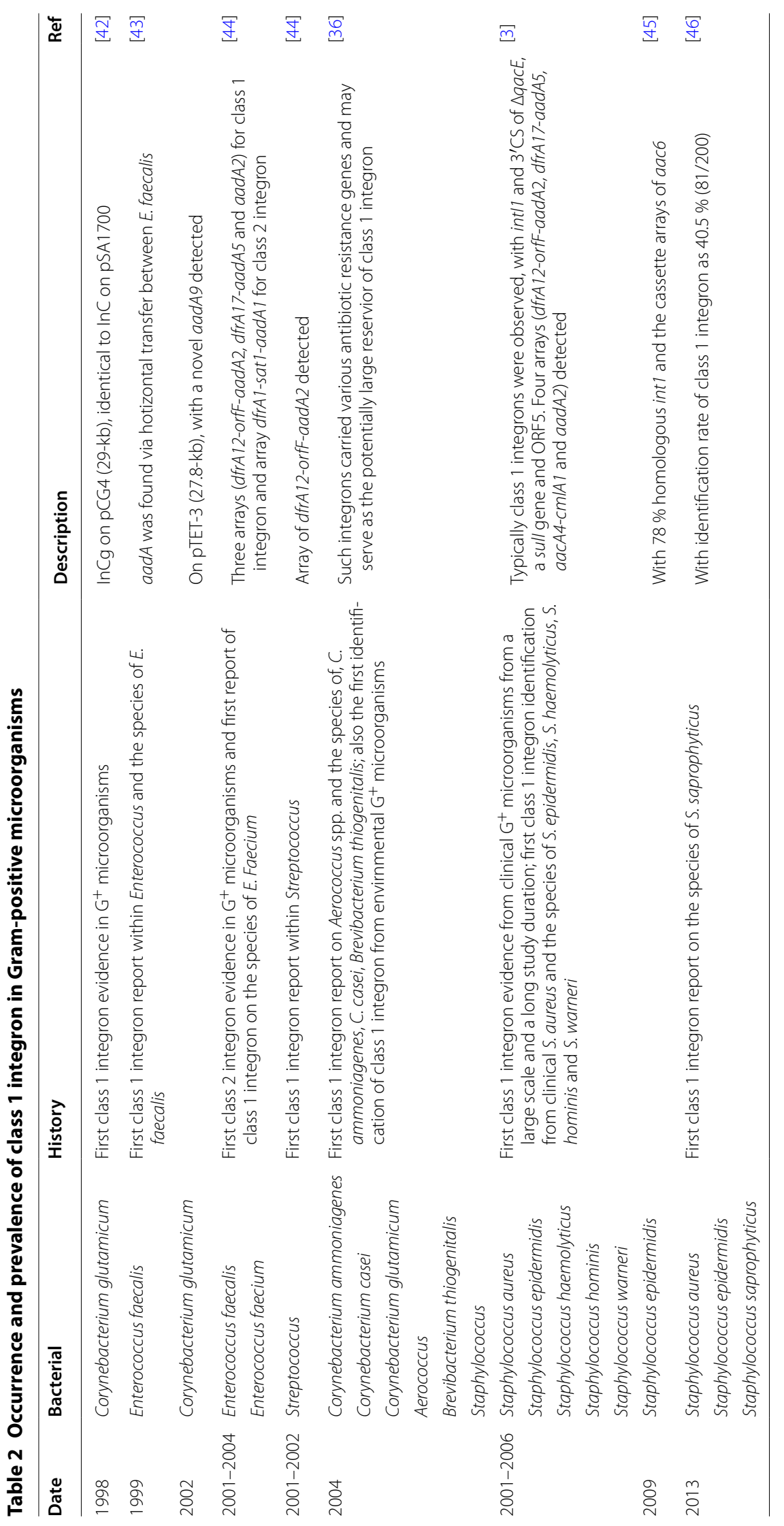


sequence element (IS1) upstream of the intI2 gene [59]. Also, a novel rearrangement of a class 2 integron ( $\operatorname{Tn} 7:$ :In2-8) with new cassettes in the variable region were recovered from 3 Acinetobacter baumannii isolates and its structure contained 6 antibiotic resistance genes within the variable region ( 3 additional genes sat 2 , aadB and catB2 inserted upstream of the 3 conventional antibiotic resistance genes of Tn 7 class 2 integron, as indicated in Table 3) [60]. In addition, the novel cassette arrays of class 2 integron ( $\operatorname{Tn} 7:: \operatorname{In} 2-1)$ was found in B. cenocepacia strain and an unusual array (sat-sat1-aadA1) in $S$. enteritidis (Table 3) [16]. The mechanism and evolution of such novel cassette arrays require further investigation and surveillance. Considered to be a major contributor to the wide spread and distribution of antibiotic resistance in microorganisms, class 2 integrons have been commonly reported in some species of Gram-negative organisms such as Acinetobacter, Enterobacteriaceae, Salmonella and Psuedomonas, with a low occurrence and prevalence comparing with class 1 integron (Table 4) [16, 17, 61-66]. From a retrospective integrons surveillance conducted in Guangzhou China during 2001-2005, class 2 integron had also been occasionally detected $(5.7 \%$, 33/583) of all tested isolates, with species of bacteria including P. aeruginosa, E. coli, E. faecalis, Proteus vulgaris and Proteus mirabilis strains, and cassettes arrays dfrA1-sat1-aadA1 obtained for all strains [19, 52].

\section{Class 3 integron}

Comparing with class 2 integron, class 3 integron contains a similar structure, as both IntII and IntI3 are part of the soil/freshwater proteobacteria group, with IntI2 found among the marine $\gamma$-proteobacteria group. Sharing similar function with IntI1, IntI3 has been identified to be capable of both catalyzing excision of integrated cassettes and integration of circularized cassettes into the attI3 site with a significantly lower recombination frequencies occurred between a 59-be and secondary sites than that observed with IntI1, and integrating various cassettes containing different attC sites into the attI3 site which was localized to a short region adjacent to intI3 [73]. This class of integron was firstly identificated from Serratia marcescens isolates in Japan in 1993, and then found to be associated with blaGES-1 from Klebsiella pneumoniae strain FFUL 22K. Its identification has been limited within a few microorganisms including Acinetobacter spp., Alcaligenes, Citrobacter freundii, Escherichia coli, Klebsiella pneumoniae, Pseudomonas aeruginosa, Pseudomonas putida, Salmonella spp and Serratia marcescens $[73,76,79,80]$ and mostly reported in low occurrence with common association with mediation IMP-1 metallo-beta-lactamase [73]. However, a class 3 integron had been lately identified containing blaGES-1 within the IncQ plasmid from E. coli [74]. The occurrence and identification rate of class 3 integron has been ranged from 0 to $10 \%$, with reports including a surveillance of 587 Gram-negative bacteria demonstrating high-level resistance to both ceftazidime and sulbactam-cefoperazone, with $0.7 \%(4 / 587)$ isolates harboring class 3 integron and an occasional report with an occurrence of $7 \%$ of veterinary isolates positive for class 3 integrase by DNA-DNA hybridization, despite discrepency when confirmed by PCR [80].

\section{Class 4 integron}

Harboring a large array of gene cassettes encoding adaptations with extension beyond antibiotic

Table 3 Summary of different structures of class 2 integrons reported in previous studies

\begin{tabular}{|c|c|c|c|c|}
\hline Name & Genes & Accession no. & Cassette arrays & Reference \\
\hline $\operatorname{Tn} 7$ & dfral-sat2-aadAl & NC_002525 & orfX 59-be tnsE tnsD tnsC tnsB tnsA & [1] \\
\hline $\operatorname{Tn} 1825$ & sat1-aadA1 & X56815 & int/2 att/2 sat1 59-be aadA1 ${ }^{59-b e}$ orfX 59-be tnsE tnsD tnsC tnsB tnsA & [48] \\
\hline Tn7::IS1-ereA & dfra1-sat1-ereA-aadA1 & AY 183453 & orfX 59-be tnsE tnsD tnsC tnsB tnsA & {$[50]$} \\
\hline AB161461 & sat-sat1-aadA1 & AB161461 & int/2 att/2 sat 59-be sat1 59-be aadA1 59-be orfX 59-be tnsE tnsD tnsC tnsB tnsA & [27] \\
\hline AB161462 & est $X$ & AB161462 & int/2 att12 dfrA159-be sat2 59-be estX 59-be aadA1 59-be orfX 59-be tnsE tnsD tnsC tnsB tnsA & [55] \\
\hline $\operatorname{Tn} 7:: \operatorname{In} 2-1$ & sat 2 & DQ082896 & int/2 atti2 sat2 59-be tnsE tnsD tnsC tnsB tnsA & {$[12]$} \\
\hline $\operatorname{Tn} 7:: \operatorname{In} 2-8$ & sat2-aadB-catB2-dfrA1-sat2-aadA1 & DQ176450 & int/2 att12 sat2 s9-be aadB 59-becatB2 att12 dfrA159-be sat2 59-beaadA1 s9-beorfX 59-be tnsE t & [51] \\
\hline
\end{tabular}


Table 4 Occurrence and prevalence of class 2, 3, and 4 integrons in Gram-positive and Gram-negative bacteria

\begin{tabular}{|c|c|c|c|}
\hline Bacterial & $\begin{array}{l}\text { Occurrence of integrons and the array of gene } \\
\text { cassettes }\end{array}$ & Sampling & Reference \\
\hline \multicolumn{4}{|l|}{ Class 2 integrons } \\
\hline Escherichia coli & $\begin{array}{l}7.4 \%(31 / 417) ; \text { dfrA1-sat2-aadA1 }(77.4 \%, 24 / 31) \\
\text { estX-sat2-aadA1 }(19.4 \%, 6 / 31) \text { and estX-sat2- } \\
\triangle \text { aadA1 }(3.2 \%, 1 / 31)\end{array}$ & BfT-GermVet monitoring study, Germany, 2004-2006 & {$[67]$} \\
\hline Enterobacteriaceae & $\begin{array}{l}34.9 \% \text { (52/149); II2 (Tn7), IIII (estX-sat2- } \\
\text { aadA1-orfX, most widely distributed) and IV2 } \\
\text { (aadA1, first reported) }\end{array}$ & $\begin{array}{l}\text { E. coli amd K. pneumoniae strains from swine } \\
\text { and chickens, Portugal }\end{array}$ & [62] \\
\hline E.coli & $3.0 \%(3 / 100)$ & Spain & [65] \\
\hline E. coli & $3.6 \%(4 / 111) ;$ dfra1-sat1-aadA1 & Preliminary study, Guangzhou, China & {$[68]$} \\
\hline E. coli & One out of 322 & $\begin{array}{l}\text { Irrigation water and associated sediments, El Paso, Presidio and } \\
\text { Weslaco }\end{array}$ & [69] \\
\hline Coliforms & $2.7 \%(5 / 183)$ & Rivers in northern region of Turkey & [63] \\
\hline $\begin{array}{r}\text { Pseudomona } \\
\text { aeruginosa }\end{array}$ & $\begin{array}{l}19.5 \%(23 / 118) \text {; dfrA1-sat1-aadA1, first report } \\
\text { of class } 2 \text { integron in this species of bacteria }\end{array}$ & Preliminary study, Guangzhou, China & {$[19]$} \\
\hline Shigella flexneri & $100 \%$ (58/58); dfrA1-sat1-aadA1 & Stool samples of sporadic diarrheic patients, China, 2005-2006 & {$[70]$} \\
\hline S. sonneii & $93 \%(2 / 43)$ & Adult patients with diarrhoea, Senegal & {$[71]$} \\
\hline S. enterica & $\begin{array}{l}85 \text { contemporary multi-drug resistant } \\
\text { D-Tartrate-Positive isolates; dfrA1-sat1-aadA1 }\end{array}$ & S. enterica Serovar Paratyphi B isolates Germany, 1995-2001 & {$[72]$} \\
\hline S. enteritidis & $4.3 \%$; estX-sat2-aadA1 & Poultry samples, Japan & {$[33]$} \\
\hline E. faecalis & $\begin{array}{l}\text { Two strains harboring Class } 1 \text { and } 2 \text { integrons; } \\
\text { dfrA } 1 \text {-sat1-aadA1, first evidence of class } 2 \text { inte- } \\
\text { gron in } \mathrm{G}^{+} \text {bacteria }\end{array}$ & Preliminary study, Guangzhou, China & {$[52]$} \\
\hline \multicolumn{4}{|l|}{ Class 3 integrons } \\
\hline E.coli & & Australia & {$[73]$} \\
\hline E.coli & ges1/oxa10:aac(6') & Switzerland & {$[74]$} \\
\hline Serratia marcescens & imp1/aacA4 & Japan & {$[75]$} \\
\hline $\begin{array}{l}\text { Klebsiella pneumo- } \\
\text { niae }\end{array}$ & ges1/oxa10:aacA4 & The urine of an intensive care unit patient in Portugal & {$[76]$} \\
\hline \multicolumn{4}{|l|}{ Class 4 integrons } \\
\hline Vibrio cholerae & & Collection de I'Institut Pasteur (CIP) & {$[77,78]$} \\
\hline V. metschnikovii & & & {$[77]$} \\
\hline
\end{tabular}

resistance and pathogenicity, class 4 integron had been firstly detected in Vibrio isolates, with its existence pre-dating the antibiotic era [77]. This distinctive class of integron had been distinguished from other RIs by two key features including both the incorporated hundreds of cassettes (For V. cholerae, at least 216 unidentified genes in an array of 179 cassettes had been identified from the cluster of VCR-associated ORFs, occupying approximate $3 \%$ of the genome) and the high homology between the attC sites of those gathered cassettes [78]. Despite its unique array of cassettes, identification of class 4 integron has been limited within microorganisms such as the Vibrionaceae, Shewanella, Xanthomonas, Pseudomonad, and other proteobacteria $[20,78,81]$. To date, class 4 integrons have been found to carry gene cassettes imparting resistance to the antibiotics chloramphenicol and fosfomycin [12].

\section{Novel perspectives in integrons} Integrons in food borne bacteria

Remaining as one of the leading concerns in public health and food safety, food-borne infections and diseases have been reported to be caused by a large variety of pathogens that contaminate food and food products. Major food borne pathogens include $S$. aureus, E. coli O157, V. parahaemolyticus, Salmonella spp. and L. monocytogenes, which are responsible for 14 million illnesses, 60,000 hospitalizations and 1800 deaths annually [19, 46-48, 8288]. Lately, indiscriminate abuse of existing antibiotics in veterinary treatment for a wide range of infectious diseases caused by bacteria in animals is found to be common, and food borne pathogens have been commonly identified from food poisoning, contamination of various food samples such as milk, pork, chicken, veal, beef, turkey and lamb meat, as well as in food production animals such as cattle, chickens, pigs and cows. As antibiotic 
resistant food borne pathogens have been considered to be a major contributor to both health-care associated and food-borne illnesses, carriage of such bacteria in a wide variety of food and food production animals are no longer limited solely to food hazard, but also poses a significant occupational risk for the industrial staff, such as handlers, asymptomatic carriers and uncolonized individuals [87]. According to our preliminary surveillance of antimicrobial resistance conducted on 96 food borne strains (including 32 Salmonella spp., 32 E. coli and 32 $S$. aureus), the phenotypic correlation existed among the aspects of antibiotic susceptibility, class 1 integrons and the abilities of biofilm formation had been firstly studied (data unpublished). In addition, class 1 integron had been discovered from food borne $S$. aureus strain, representing the first evidence of class 1 integron from food borne Gram-positive microorganisms as Staphylococcus. This novel finding may offer significant guidance in effective control on dissemination of antibiotic resistance of foodborne pathogens, nevertheless, the occurrence and prevalence of integrons, including class 1 integron and other classes of integrons, as well as the role of such integrons play in the antimicrobial resistance in food safety, require further investigation.

\section{Concluding remarks}

Antimicrobial resistance still remains the leading concern in global public health and food safety, as bacteria are capable of obtaining resistance gene through either genetic mutation or horizontal transfer of resistance genes. Horizontal transfer of resistance genes are considered to be the major cause to facilitate the rapid spread of antibiotic resistance in microbes. As a frequently reported resistance mechanism served as horizontal transfer among microbes and found to be a common genetic element existed in $9 \%$ of bacteria and representatives from a broad range of phyla and environments, integrons play core role in antibiotic resistance of microorganisms and have been shown to contribute to the wide spread and distribution of antibiotic resistant genes among bacteria, as well as the bacterial evolution and adaption [89]. The currently available studies and investigations have been restricted and limited within class 1 integron with perspectives on Gram-negative bacteria. Nevertheless, class 1 integron on Gram-positive microorganisms, together with class 2, 3 and 4 integrons has barely been touched upon, making such concerns potentially be unnoticed and neglected antibiotic resistance determinants. As consequence, identification of integrons regarding the species of involved microorganisms, occurrence and prevalence of different classes of integrons in certain species of bacteria, distribution and spread of integrons and cassettes arrays, as well as the role of such integrons play in the dissemination and spread of antimicrobial resistance, require further investigation.

\section{Authors' contributions}

YD participated in the design of the review, summary of data and drafted the manuscript. XB carried out the further data collection of integrons, preparation of the figures and tables, as well as the revision of the manuscript. $L$ performed the data analysis. $L C$ and $J L$ carried out the summary of integrons data and processing of figures and tables. MJ and DC performed the statistical analysis and revision. YL participated in the design of the study, draft and revision the manuscript. GY conceived of the study and participated in its design and coordination. All authors read and approved the final manuscript.

\section{Author details}

${ }^{1}$ College of Light Industry and Food Sciences, South China University of Technology, Guangzhou 510640, China. ${ }^{2}$ Institute of Agro-products Processing, Anhui Academy of Agricultural Sciences, Hefei 230031, China. ${ }^{3}$ Department of Laboratory Medicine, First Affiliated Hospital of Guangzhou Medical College, Guangzhou 510120, China. ${ }^{4}$ The Third Affiliated Hospital of Sun Yat-sen University, Guangzhou 510630, China. ${ }^{5}$ Guangzhou Women and Children's Medical Center, 9 Jinsui Road, Guangzhou 510620, China. ${ }^{6}$ First Affiliated Hospital of Jinan University, Guangzhou 510620, China.

\section{Acknowledgements}

This work was supported by the National 973-Plan of China (2012CB720800), International Science and Technology Cooperation Program from Science and Technology Planning Project of Guangdong Province, China (Dr. Bing Li, 2013-2016), National Natural Science Foundation of China (31201362 and 31101278), the National Science and Technology Support Program (2012BAD37B01), National Outstanding Doctoral Dissertation Funding (D4140010), Guangdong Outstanding Doctoral Dissertation Funding (K3140030) and the Fundamental Research Funds for the Central Universities (2012ZM0060)

\section{Competing interests}

The authors declare that they have no competing interests.

Received: 26 February 2015 Accepted: 14 August 2015 Published online: 20 October 2015

\section{References}

1. Hussein AlA, Ahmed AM, Sato M, Shimamoto T (2009) Characteriaztion of integrons and antimicrobial resistance genes in clinical isolates of Gram-negative bacteria from Palestinian hospitals. Microbiol Immunol 53:595-602

2. Xu Z, Li L, Shi L, Shirtliff M (2011) Class 1 integron in staphylococci. Mol Biol Rep 38:5261-5279

3. Xu Z, Li L, Shirtliff M, Peters B, Li B, Peng Y, Alam M, Yamasaki S, Shi L (2011) Resistance class 1 integron in clinical methicillin-resistant Staphylococcus aureus strains in southern China, 2001-2006. Clin Microbiol Infect $17: 714-717$

4. Zhong N, Gui Z, Xu L, Huang J, Hu K, Gao Y, Zhang X, Xu Z, Su J, Li B (2013) Solvent-free enzymatic synthesis of 1,3-diacylglycerols by direct esterification of glycerol with saturated fatty acids. Lip Heal Dis 12:65-72

5. You R, Gui Z, Xu Z, Shirtliff M, Yu G, Zhao X, Shi L, Li B, Su J, Li L (2012) Methicillin-resistance Staphylococcus aureus detection by an improved rapid PCR assay. Afr J Microbiol Res 6:7131-7133

6. Hall RM, Collis CM (1995) Mobile gene cassettes and integrons: capture and spread of genes by site-specific recombination. Mol Microbiol 15:593-600

7. Mazel D (2006) Integrons: agents of bacterial evolution. Nat Rev Microbiol 4:608-620

8. Xu Z, Li L, Zhao X, Chu J, Li B, Shi L, Su J, Shirtliff M (2011) Development and application of a novel multiplex polymerase chain reaction (PCR) assay for rapid detection of various types of staphylococci strains. Afr J Microbiol Res 5:1869-1873 
9. Xu Z, Liu X, Li L, Li B (2013) Development of Staphylococcus aureus enterotoxin in food borne bacteria. Mod Food Sci 29:2317-2324

10. Stokes HW, Hall RM (1989) A novel family of potentially mobile DNA elements encoding site-specific gene-integration functions: integrons. Mol Microbiol 3:1669-1683

11. Hall RM, Collis CM, Kim MJ, Partridge SR, Recchia GD, Stokes HW (1999) Mobile gene cassettes and integrons in evolution. Ann NY Acad Sci 870:68-80

12. Fluit AC, Schmitz FJ (2004) Resistance integrons and super-integrons. Clin Microbiol Infect 10:272-288

13. Boucher Y, Labbate M, Koenig JE, Stokes HW (2007) Integrons: mobilizable platforms that promote genetic diversity in bacteria. Trends Microbiol 15:301-309

14. Partridge SR, Tsafnat G, Coiera E, Iredell JR (2009) Gene cassettes and cassette arrays in mobile resistance integrons. FEMS Microbiol Rev 33:757-784

15. Francia MV, Zabala JC, de la Cruz F (1999) Garcia Lobo JM: the Int/1 integron integrase preferentially binds single stranded DNA of the attC site. J Bacteriol 181:6844-6849

16. Ramírez MS, Vargas LJ, Cagnoni V, Tokumoto M (2005) Class 2 integron with a novel cassette array in a Burkholderia cenocepacia isolate. Antimicrob Agents Chemother 49:4418-4420

17. Crowley D, Cryan B, Lucey B (2008) First detection of a class 2 integron among clinical isolates of Serratia marcescens. Br J Biomed Sci 65:86-99

18. Stokes HW, O'Gorman DB, Recchia GD, Parsekhian M, Hall RM (1997) Structure and function of 59-base element recombination sites associated with mobile gene cassettes. Mol Microbiol 26:731-745

19. Xu Z, Li L, Shirtliff M, Alam M, Yamasaki S, Shi L (2009) Occurrence and characteristics of class 1 and 2 integrons in Pseudomonas aeruginosa isolates from patients in southern China. J Clin Microbiol 47:230-234

20. Rowe-Magnus DA, Mazel D (2001) Integrons: natural tools for bacterial genome evolution. Curr Opin Microbiol 4:565-569

21. Cambray G, Guerout AM, Mazel D (2010) Integrons. Annu Rev Genet 44:141-166

22. Mazel D (2006) Integrons: agents of bacterial evolution. Nat Rev Microbiol 4:608-620

23. Tauch A, Gotker S, Puhler A, Kalinowski J, Thierbach G (2002) The 27.8-kb R-plasmid PTET3 from Corynebacterium glutamicum encodes the aminoglycoside adenyltransferase gene cassette aadA9 and the regulated tetracycline efflux system Tet 33 flanked by active copies of the widespread insertion sequence IS6100. Plasmid 48:117-129

24. Nemergut DR, Robeson MS, Kysela RF, Martin AP, Schmidt SK, Knight R (2008) Insights and inferences about integron evolution from genomic data. BMC Genom 9:1-12

25. Collis CM, Grammaticopoulos G, Briton J, Stokes HW, Hall RM (1993) Site-specific insertion of gene cassettes into integrons. Mol Microbiol 9:41-52

26. Barker A, Clark CA, Manning PA (2002) Identification of VCR, a repeated sequence associated with a locus encoding a hemagglutinin in Vibrio cholerae 01. J Bacteriol 176:5450-5458

27. Martín BS, Lapierre L, Cornejo J, Bucarey S (2008) Characterization of antibiotic resistance genes linked to class 1 and 2 integrons in strains of Salmonella spp. isolated from swine. Can J Microbiol 54:569-576

28. Nield BS, Holmes AJ, Gillings MR, Recchia GD, Mabbutt BC, Nevalainen KM, Stoke HM (2001) Recovery of new integron classes from environmental DNA. FEMS Microbiol Lett 195:59-65

29. Barlow RS, Desmarchelier PM, Gobius KS (2004) Isolation and characterization of integron-containing bacteria without antibiotic selection. Antimicrob Agents Chemother 48:838-842

30. Labbate M, Case RJ, Stokes HW (2009) The integron/gene cassette system: an active player in bacterial adaptation. Methods Mol Biol 532:103-125

31. Recchia GD, Hall RM (1997) Origins of the mobile gene cassettes found in integrons. Trends Microbiol 5:389-394

32. Abigial PV, Elizabeth FR, Everardo CQ (2009) Detection and characterization of class 1 integrons in Aeromonas spp. isolated from human diarrheic stool in Mexico. J Basic Microbiol 49:572-578

33. Ahmed AM, Nakano H, Shimamoto T (2002) Molecular characterization of integrons in non-typhoid Salmonella serovars isolated in Japan: description of an unusual class 2 integron. J Antimicrob Chemother 55:371-374
34. Ahmed AM, Furuta K, Shimomura K, Kasama Y, Shimamoto T (2006) Genetic characterization of multidrug resistance in Shigella spp. from Japan. J Med Microbiol 55:1685-1691

35. Ahmed AM, Ishida Y, Shimamoto T (2009) Molecular characterization of antimicrobial resistance in Salmonella isolated from animals in Japan. J Appl Microbiol 106:402-409

36. Chang LL, Chen HF, Chang CY, Lee TM, Wu WJ (2004) Contribution of integrons, and SmeABC and SmeDEF efflux pumps to multidrug resistance in clinical isolates of Stenotrophomonas maltophilia. J Antimicrob Chemother 53:518-521

37. O'Halloran F, Lucey B, Cryan B, Buckley T, Fanning S (2004) Molecular characterization of class 1 integrons from Irish thermophilic Campylobacter spp. J Antimicrob Chemother 53:952-957

38. Crowley D, Daly M, Lucey B, Shine P, Collins JJ, Cryan B, Moore JE, Murphy P, Buckley G, Fanning S (2002) Molecular epidemiology of cystic fibrosislinked Burkholderia cepacia complex isolates from three national referral centres in Ireland. J Appl Microbiol 92:992-1004

39. Dalsgaard A, Forslund A, Serichantalergs O, Sandvang D (2000) Distribution and content of class 1 integrons in different Vibrio cholerae O-serotype strains isolated in Thailand. Antimicrob Agents Chemother 44:1315-1321

40. Fereshteh S, Farzad B, Mohammad MF (2010) Molecular characterization of class 1 integrons in MDR Pseudomonas aeruginosa isolated from clinical settings in Iran, Tehran. FEMS Immunol Med Microbiol 58:421-425

41. Huang SC, Chiu CH, Chiou CS, Yang YJ (2013) Multidrug-resistant Salmonella enterica serovar Panama carrying class 1 integrons is invasive in Taiwanese children. J Formos Med Assoc 112:269-275

42. Marianne S (2005) Prevalence and characterization of class 1 and class 2 integrons in Escherichia coli isolated from meat and meat products of Norwegian origin. J Antimicrob Chemother 56:1019-1024

43. Nandi S, Maurer JJ, Hofacre C, Summers AO (2004) Gram-positive bacteria are a major reservoir of class 1 antibiotic resistance integrons in poultry litter. Proc Natl Acad Sci USA 101:7118-7122

44. Nikokar I, Tishayar A, Flakiyan Z, Alijani K, Rehana-Banisaeed S, Hossinpour M, Amir-Alvaei S, Araghian A (2013) Antibiotic resistance and frequency of class 1 integrons among Pseudomonas aeruginosa, isolated from burn patients in Guilan, Iran. Iran J Microbiol 5:36-41

45. Partridge SR, Collis CM, Hall RM (2002) Class 1 integron containing a new gene cassette, aadA10, associated with Tn1404 from R151. Antimicrob Agents Chemother 46:2400-2408

46. Xu Z, Shi L, Zhang C, Zhang L, Li X, Cao Y, Li L, Yamasaki S (2007) Nosocomial infection caused by class 1 integron-carrying Staphylococcus aureus in a hospital in South China. Clin Microbiol Infect 13:980-984

47. Xu Z, Li L, Alam M, Yamasaki S, Shi L (2008) First confirmation of integronbearing methicillin-resistant Staphylococcus aureus. Curr Microbiol 57:264-268

48. Xu Z, Shi L, Alam M, Li L, Yamasaki S (2008) Integron-bearing methicillinresistant coagulase-negative staphylococci in South China, 2001-2004. FEMS Microbiol Lett 278:223-230

49. Deng Y, Liu J, Peters B, Chen D, Yu G, Xu Z, Shirtliff M (2015) Antimicrobial resistance investigation on Staphylococcus Strains in a local Hospital in Southern China, 2001-2010. Microb Drug Resist 21:102-104

50. Nesvera J, Hochmannová J, Patek M (1998) An integron of class 1 is present on the plasmid pCG4 from Gram-positive bacterium Corynebacterium glutamicum. FEMS Microbiol Lett 169:391-395

51. Clark NC, Olsvik $\varnothing$, Swenson JM, Spiegel CA, Tenover FC (1999) Detection of a streptomycin/spectinomycin adenylyltransferase gene (aadA) in Enterococcus faecalis. Antimicrob Agents Chemother 43:157-160

52. Xu Z, Li L, Shirtliff M, Peters B, Peng Y, Alam M, Yamasaki S, Shi L (2010) First report of class 2 integron in clinical Enterococcus faecalis and class 1 integron in Enterococcus faecium in South China. Diag Microbiol Infect Dis 68:315-317

53. Xu Z, Gui Z, Zhao X, Zhang Y, He X, Li W, Yang L (2012) Expression and purification of gp41-gp36 fusion protein and application in serological screening assay of HIV-1 and HIV-2. Afr J Microbiol Res 6:6295-6299

54. Pinilla G, Muñoz L, Ruiz A, Chavarro B, Cifuentes Y (2009) Isolation of Staphylococcus epidermidis strain carrier of the class one integron in a septic neonatal patient. Infectio 13:196-202

55. Veise P, Ramazanzadeh R, Khiababi Z, Derakhshi B, Amirmozafari N (2013) Identification of class I integrons gene in Staphylococcus strains isolated from clinical samples. Cell Biol 1:24-27 
56. Senda K, Arakawa Y, Ichiyama S, Nakashima K, Ito H, Ohsuka S, Shimokata K, Kato N, Ohta M (1996) PCR detection of metallo-beta-lactamase gene (blalMP) in gram-negative rods resistant to broad-spectrum beta-lactams. J Clin Microbiol 34:2909-2913

57. Hansson K, Sundström L, Pelletier A, Roy PH (2002) Intl2 integron integrase in Tn7. J Bacteriol 184:1712-1721

58. Barlow RS, Gobius KS (2006) Diverse class 2 integrons in bacteria from beef cattle sources. J Antimicrob Chemother 58:1133-1138

59. Biskri L, Mazel D (2003) Erythromycin esterase gene ere(A) is located in a functional gene cassette in an unusual class 2 integron. Antimicrob Agents Chemother 47:3326-3331

60. Ramírez MS, Quirogaand C, Centrón D (2005) Novel rearrangement of a class 2 integron in two non-epidemiologically related isolates of Acinetobacter baumannii. Antimicrob Agents Chemother 49:5179-5181

61. Macedo-Viñas M, Cordeiro NF, Bado I, Herrera-Leon S, Vola M, Robino L, Gonzalez-Sane R, Mateos S, Schelotto F, Algorta G, Ayala JA, Echeita A, Vignoli R (2009) Surveillance of antibiotic resistance evolution and detection of class 1 and 2 integrons in human isolates of multi-resistant Salmonella Typhimurium obtained in Uruguay between 1976 and 2000. Int J Infect Dis 13:342-348

62. Machado E, Coque TM, Cantón R, Sousa JC, Peixe L (2008) Antibiotic resistance integrons and extended-spectrum $\beta$-lactamases among Enterobacteriaceae isolates recovered from chickens and swine in Portugal. J Antimicrob Chemother 62:296-302

63. Ozgumus OB, Sandalli C, Sevim A, Celik-Sevim E, Sivri N (2009) Class 1 and class 2 integrons and plasmid-mediated antibiotic resistance in coliforms isolated from ten rivers in northern Turkey. J Microbiol 47:19-27

64. Solberg OD, Ajiboye RM, Riley LW (2006) Origin of class 1 and 2 integrons and gene cassettes in a population-based sample of uropathogenic Escherichia coli. J Clin Microbiol 44:1347-1351

65. Vinué L, Sáenz Y, Somalo S, Escudero E, Moreno MA, Ruiz-Larrea F, Torres C (2008) Prevalence and diversity of integrons and associated resistance genes in faecal Escherichia coli isolates of healthy humans in Spain. J Antimicrob Chemother 62:934-937

66. Xu H, Broersma K, Miao V, Davies J (2011) Class 1 and class 2 integrons in multidrug-resistant gram-negative bacteria isolated from the Salmon River, British Columbia. Can J Microbiol 57:460-470

67. Kadlec K, Schwarz S (2008) Analysis and distribution of class 1 and class 2 integrons and associated gene cassettes among Escherichia coli isolates from swine, horses, cats and dogs collected in the BfT-GermVet monitoring study. J Antimicrob Chemother 62:469-473

68. Su J, Shi L, Yang L, Xiao Z, Li X, Li L, Yamasaki S (2006) Analysis of integrons in clinical isolates of Escherichia coli in China during the last six years. FEMS Microbiol Lett 254:75-80

69. Roe MT, Vega E, Pillai SD (2003) Antimicrobial resistance markers of class 1 and class 2 integronbearing Escherichia coli from irrigation water and sediments. Emerg Infect Dis 9:822-826

70. Zhu JY, Duan GC, Yang HY, Fan QT, Xi YL (2011) Atypical class 1 integron coexists with class 1 and class 2 integrons in multi-drug resistant Shigella flexneri isolates from China. Curr Microbiol 62:802-806

71. Gassama-Sow A, Diallo MH, Boye CS, Garin B, Sire JM, Sow Al, Aïdara-Kane A (2006) Class 2 integron-associated antibiotic resistance in Shigella sonnei isolates in Dakar, Senegal. Int J Antimicrob Agents 27:267-270

72. Miko A, Pries K, Schroeter A, Helmuth R (2003) Multiple-drug resistance in D-tartrate-positive Salmonella enterica serovar paratyphi B isolates from poultry is mediated by class 2 integrons inserted into the bacteria chromosome. Antimicrob Agents Chemother 47:3640-3643

73. Arakawa Y, Murakami M, Suzuki K, Ito H, Wacharotayankun R, Ohsuka S, Kato N, Ohta M (1995) A novel integron-like element carrying the metallo- $\beta$-lactamase gene bla ${ }_{\text {IMP }}$. Antimicrob Agents Chemother 39:1612-1615

74. Collis CM, Kim MJ, Partridge SR, Stokes HW, Hall RM (2002) Characterization of the class 3 integron and the site-specific recombination system it determines. J Bacteriol 84:3017-3126

75. Correia M, Boavida F, Grosso F, Salgado MJ, Lito LM, Cristino JM, Mendo S, Duarte A (2003) Molecular characterization of a new class 3 integron in Klebsiella pneumoniae. Antimicrob Agents Chemother 47:2838-2843
76. Ploy MC, Chainier D, Tran Thi NH, Poilane I, Cruaud P, Denis F, Collignon A, Lambert T (2003) Integron-associated antibiotic resistance in Salmonella enterica serovar typhi from Asia. Antimicrob Agents Chemother 47:1427-1429

77. Shibata N, Doi Y, Yamane K, Yagi T, Kurokawa H, Shibayama K, Kato H, Kai K, Arakawa Y (2003) PCR typing of genetic determinants for metallo-betalactamases and integrases carried by gram-negative bacteria isolated in Japan, with focus on the class 3 integron. J Clin Microbiol 41:5407-5413

78. Poirel L, Carattoli A, Bernabeu S, Bruderer T, Frei R, Nordmann P (2010) A novel IncQ plasmid type harbouring a class 3 integron from Escherichia coli. J Antimicrob Chemother 65:1594-1598

79. Rowe-Magnus DA, Guerout AM, Mazel D (1999) Super-integrons. Res Microbiol 150:641-651

80. Rowe-Magnus DA, Guerout AM, Ploncard P, Dychinco B, Davies J, Mazel D (2001) The evolutionary history of chromosomal super-integrons provides an ancestry for multiresistant integrons. Proc Natl Acad Sci USA 98:652-657

81. Clark CA, Purins L, Kaewrakon P, Focareta T, Manning PA (2000) The Vibrio cholerae $\mathrm{O} 1$ chromosomal integron. Microbiol 146:2605-2612

82. Wang L, Li Y, Xu Z, Zhong Q (2012) Development and application of a simple loop-mediated isothermal amplification method on rapid detection of Listeria monocytogenes strains. Mol Biol Rep 39:445-449

83. Zhao X, Li Y, Wang L, You L, Xu Z, Li L, He X, Liu Y, Wang J, Yang L (2010) Development and application of a loop-mediated isothermal amplification method on rapid detection Escherichia coli 0157 strains from food samples. Mol Biol Rep 37:2183-2188

84. Zhao X, Wang L, Chu J, Li Y, Li Y, Xu Z, Li L, Shirtliff M, He X, Liu Y, Wang J, Yang $L$ (2010) Development and application of a rapid and simple loopmediated isothermal amplification method for food-borne Salmonella detection. Food Sci Biotechnol 19:1655-1659

85. Zhao X, Wang L, Chu J, Li Y, Li Y, Xu Z, Li L, Shirtliff M, He X, Liu Y, Wang $J$, Yang L (2010) Rapid detection of Vibrio parahaemolyticus strains and virulent factors by loop-mediated isothermal amplification assays. Food Sci Biotechnol 19:1191-1197

86. Zhao X, Wang L, Li Y, Xu Z, Li L, He X, Liu Y, Wang J, Yang L (2011) Development and application of a loop-mediated isothermal amplification method on rapid detection of Pseudomonas aeruginosa strains. World J Microbiol Biotechnol 27:181-184

87. Xu Z, Li L, Chu J, Peters B, Harris M, Li B, Shi L, Shirtliff M (2012) Development and application of loop-mediated isothermal amplification assays on rapid detection of various types of staphylococci strains. Food Res Int 47:166-173

88. Deng Y, Liu C, Li B, Li L, Xu Z (2015) Review of methicillin-resistant Staphylococcus aureus and its detection in food safety. Mod Food Sci 31:259-266

89. Sundström $L$ (1998) The potential of integrons and connected programmed rearrangements for mediating horizontal gene transfer. APMIS Suppl 84:37-42

\section{Submit your next manuscript to BioMed Central and take full advantage of:}

- Convenient online submission

- Thorough peer review

- No space constraints or color figure charges

- Immediate publication on acceptance

- Inclusion in PubMed, CAS, Scopus and Google Scholar

- Research which is freely available for redistribution

Submit your manuscript at

www.biomedcentral.com/submit

C BioMed Central 